RESEARCH ARTICLE

\title{
An Approach to Indexical Beliefs
}

\author{
Tadeusz Ciecierski*
}

Received: 11 June 2019 / Accepted: 3 February 2020

\begin{abstract}
An approach to indexical beliefs is presented and defended in the paper. The account is inspired by David Kaplan's representationalist analysis of de re belief reports. I argue that imposing additional constraints on the Kaplanian notion of representation results in an elegant theory of indexical beliefs. The theory is committed to representations of limited accessibility but is not committed to relativized proposition, special de se contents or propositions of limited accessibility.
\end{abstract}

Keywords: Belief de hic; belief de nunc; belief de se; indexical beliefs; propositions; representations.

The problem of indexical attitudes in general and de se attitudes in particular is the subject matter of vivid discussion in philosophy and linguistics. Some philosophers suggested even that addressing the problem properly requires the modification of the standard notion of truth-conditional content or demands a new theory of objects of attitudes. Without any doubt, the realization of any of the two expectations would have an enormous impact on numerous philosophical considerations regarding the nature of agents, actions, intentionality, and reference. This promise (for

* University of Warsaw

(iD) https://orcid.org/0000-0001-5403-9590

Institute of Philosophy, University of Warsaw, Krakowskie Przedmieście 3, 00-927 Warsaw, Poland

$\triangle$ taci@uw.edu.pl

(C) The Author. Journal compilation (c) The Editorial Board, Organon F.

This article is distributed under the terms of the Creative Commons Attribution-NonCommercial 4.0 International Public License (CC BY-NC 4.0). 
some) or threat (for others) suffices for the justification of the relevance of the problem in question. That having been said, however, it is difficult sometimes to say what the exact problem of indexical attitudes is as there are numerous issues discussed in the literature under this heading. It might be useful, therefore, to start with a short classification of the problems in questions. Following (Higginbotham 2003) we may start with the list of the following four questions: ${ }^{1}$

Problem 1. What is the nature of essentially indexical interpretations of certain sentences or utterances?

Problem 2. What relation do they bear to ordinary uses of the firstperson pronoun, adverbs of time (like 'now') and place (like 'here')?

Problem 3. Why are they triggered by the particular linguistic items that trigger them?

Problem 4. Are they universal in human language, and what relation, if any, do they bear to logophoric phenomena?

and add to the list the following problem:

Problem 5. What is the nature of essentially indexical attitudes?

Below I shall be interested solely in problems 1, 2 and 5 (by "the problem of indexical beliefs" I shall mean below nothing more than the three problems in question).

My general assumption here would be that the study of attitude reports or particular readings of such reports sheds light on the nature of corresponding attitudes. This assumption, I believe, is often made in the literature but rarely stated explicitly. ${ }^{2}$ Consider any true attitude report of the form "A believes that p." It is natural to ask a question in virtue of which non-linguistic facts the report is true (or - to use fashionable terminology of truthamkers: which non-linguistic facts make the report true). The immediate answer to this question is that the report is true in virtue of the fact that $\mathrm{A}$ is in a certain kind of mental state. It is, of course, a matter of the

1 Higginbotham's formulation of the problems concerns de se beliefs only but they might be easily generalized to other essentially indexical cases.

2 For some exceptions, see (Vendler 1967; Peterson 1997; and Ciecierski 2016). 
debate whether the state can be characterized solely internally or requires an appeal to external factors but this should not obscure the fact that the existence of the state in question is a necessary condition of the report being true. This general point is accepted even by eliminativists who deny not the connection between the existence of the state and truthfulness of the report but the fact that there are at all states of a certain kind (and hence: that the attitude reports have truth values at all). Now the natural next question is: "How the utterance selects, out of a vast universe of possible states of affairs, the ones that are potential truthmakers of the report?" It goes without saying that the answer must appeal to semantical properties of "A believes that p." The semantic properties, whatever they are (this, again, is a matter of vivid debates in philosophy), point out at generic features that each potential truthmaker of the report must be entitled with. This is how the assumption that the study of attitude reports reveals properties of the corresponding attitudes will be understood in this paper.

The purpose of this paper is to present and defend a relatively conservative approach to indexical beliefs and indexical belief reports. It shall be conservative as it requires no changes in our theories of truth-conditional content. It shall not be completely conservative as it denies that propositions are objects of attitudes. However, it shall still be relatively conservative as the direct objects of attitudes are familiar entities: mental representations. The plan of the paper looks as follows. I shall start with a brief recapitulation of the problem of indexical beliefs. Next, I shall describe an account in which I will offer a theoretical description of the phenomenon. Finally, I shall address several matters that, I think, may help us to better understand the scope and possible limits of the account. ${ }^{3}$

3 Below I shall use terms such as "indexical belief" (Stalnaker 1981), "locating belief" (Perry 1979), "self-locating belief" (Lewis 1979; and Perry 2013) and "essentially indexical belief" (Stalnaker 1981 again) interchangeably. The terms such as "de se belief," "de nunc belief" and "de hic belief", on the other hand, will be used to refer to the proper subsets of the class of locating beliefs. I am not presupposing that the subsets are (or are not) disjoint.

Organon F 27 (3) 2020: 346-376 


\section{Preliminaries}

What are indexical beliefs? The standard answer to this question is that they are beliefs about "[...] where one is, when it is, and who one is" (Perry 1979, 5). ${ }^{4}$ This answer is usually supplemented with scenarios that are intended to make the essentially indexical character of such beliefs explicit.

Consider a belief report:

(1) A believes that she is F.

This report might be true in two radically different situations: one in which $\mathrm{A}$ is aware of the fact that she herself is $\mathrm{F}$ and the other where she is unaware of that. The two situations might be more precisely referred to by means of the following paraphrases or readings of $(1):^{5}$

(2) A believes that she herself is F.

(3) A believes that she (but not that she herself) is F.

Some suggest (cf. Jaszczolt 2013) that (2) and (3) are distinct interpretations of (1), and that this means that (1) is context dependent. Others may perhaps think that (1) is ambiguous. Yet others may be willing to treat (1) as a manifestation of the phenomenon of generality, that is the fact that

4 A slightly different characteristic is provided in (Perry 2013): "A belief is selflocating if the truth of the belief constraints the location of the belief and the believer" (Perry 2013, 388). One may also explain the notion of indexical belief by saying that it is a belief state naturally expressed by 'I'-sentences, 'now'-sentences or 'here'-sentences (thanks to the anonymous reviewer for this suggestion). This intuitive description, however, is rather unclear as it adduces to the very imprecise concept of naturalness of expression.

5 If one thinks that constructions like '(she) herself' do not univocally specify de se attributions (cf. Schlenker 2011), then the point is that there is a difference in truth conditions of counterparts of (2) and (3) that, respectively, contain or do not contain, elements that trigger de se attributions. Self-oriented long distance reflexives of Sells (1987) and Chierchia (1989) might be good candidates for such triggering elements. Let me just stress that nothing in this paper depends on the theoretical choice on that matter. 
a meaning or a connotation of the expression is disjunctive. ${ }^{6}$ The choice of the particular account here constitutes an answer to the Problem 3 and lies beyond the scope of this paper. ${ }^{7}$ The common assumption of the alternative analyzes is that (2) and (3) differ in truth conditions.

This common assumption consists in the claim that (2) and (3) are true of situations that involve distinct kinds of belief states. This difference manifests in the dispositions and actions of the agent: if one accepts a functionalist picture of beliefs, one probably has to admit that the two belief states occupy different places in the functional web of beliefs, desires and actions. Philosophers believing in intentional explanations and predictions will notice that the regular intentional predictions in order to have any predictive and explanatory value must involve premises that have an indexical interpretation. Otherwise nothing interesting about agent's actions can be inferred. ${ }^{8}$ This shows that indexical beliefs are ubiquitous as well as that the notion of indexical belief plays important role in practices of action explanation and prediction. ${ }^{9}$

${ }^{6} \quad$ For a useful discussion about differences between ambiguity, indeterminacy and generality see (Gillon 1990).

7 That being said, however, I would like to stress similarities between certain consequences of the present theory and the pragmatic theories that address the Problem 3. On the account defended in this paper, for instance, indexical attitudes are always attitudes de re. This analysis fits well the pragmatic theories (see Capone 2016) that make use of the indexical-de re entailment in order to explain the default character of certain (de se) readings in terms of scalar implicatures based on the de se-de re entailment scale.

8 As John Perry once noted: when looking at our actions from the first-person perspective we quickly realize that: "When we replace $\langle\langle\mathrm{I}\rangle\rangle$ with other designations of me, we no longer have an explanation of my behavior and so, it seems, no longer attribution of the same belief" (Perry 1979, 3). This justifies, as Perry observes, classifying such beliefs as essentially indexical. As suggested by authors like Castañeda (Castañeda 1966) and Corazza (cf. Corazza 2004, 275-307) even a stronger claim holds: they are essentially thusly indexical as they involve a peculiar type of indexicality or mode of reference.

$9 \quad$ It should be noted, however, that this broadly accepted belief has been questioned in recent years by authors like Cappelen and Dever (cf. Cappelen and Dever 2013). 
It is also common to claim that indexical beliefs are philosophically interesting because they seem to put in question a popular picture of propositional intentional states. According to this popular picture (call it the relationists view) propositional attitudes are two-argument relations ${ }^{10}$ that link cognitive subjects to abstract objects called "propositions." The relationists view assumes that propositions are truth evaluable entities that are eternally, impersonally and placelessly (and, in general, contextlessly) true or false. It embraces also the view that propositions are universally accessible.

What is the challenge posed by indexical beliefs? Consider the following scenario:

\section{Absent-minded referee}

Suppose that Monica received a paper to review. Monica is unaware that she is in fact its author (she might have submitted it a very long time ago and forgot about it). At a certain point she concludes that the paper should be rejected. Later she may (or she may not) realize that she in fact negatively evaluated her own work.

There can be no doubt that Monica's behavioral potential would have changed dramatically if she had acquired the indexical belief that she herself is the author of the paper. According to the view that combines relationism with anti-relativism the difference in the behavioral potential ought to be explained by the fact that in the two situations Monica is in the believing relation towards distinct propositions. Call the propositional content of indexical belief (whatever it is) an indexical proposition. Two options are prima facie available here: the indexical proposition is either general or singular. ${ }^{11}$ However, both options are rather implausible. Monica might have

10 Strictly speaking, relationism may enable more arguments in belief relation (e.g. time or place). The important point is that the additional arguments are not essentially linked to other representational properties of the cognitive agent.

11 One might object to this description as committed to the false dilemma that ignores the possibility of singular contents of a special kind (special first-person de re senses, for instance). However, the commitment to such special de re senses conflicts with the assumption of universal accessibility. Hence such a theory is in conflict with relationism and anti-relativism. 
already believed the general proposition that makes use of some descriptive concept D that uniquely refers to her. For instance, she may be the tallest woman ever born in Palo Alto and she might have been related to the proposition that she had rejected the paper authored by the tallest woman ever born in Palo Alto. Having such (or similar) belief does not preclude a huge change in her behavioral potential when she acquires the indexical belief. The only additional condition that has to be met is that she did not know earlier that she is D. The same seems to apply to singular propositions: she clearly might have believed that she rejected the paper authored by $\mathrm{N}$ (where $\mathrm{N}$ is a non-first person, directly referential term referring to her) before and still be the subject of the relevant belief expansion. Again, the only additional condition that has to be met is that she didn't know that she is $\mathrm{N}$.

So, it seems that the combination of relationism and anti-relativism is unable to explain the relevant change in beliefs. Some authors (e.g. Feit and Capone 2013) call this issue "the psychological problem of de se." The challenge here is to "[...] identify the content of a given de se belief" and to distinguish it from contents of beliefs that are not de se (Feit and Capone 2013,3 ). Additionally, they single out the linguistic problem of de se (what is the semantic value of "I," "s/he herself" or self-oriented long distance reflexives in belief clauses; the problem is a reformulation of Higginbotham's Problem 2) and the generality problem identified by Geach ("How distinct people can share the same de se belief?"). Geach's problem is closely connected with the problem of making sense of the intuitive assumption that distinct agents might perform the same type of action (Verdejo 2017). Indeed, if one dismisses the presupposition of Geach's question (namely that distinct people can share the same de se belief) it may be difficult to make sense of sameness of action that are - due to the involvement of de se attitudes - agent bound. In the section 3 below I shall discuss how the theory sketched below deals with the three problems. ${ }^{12}$

12 In this paper I am not discussing problems that arise if a particular view about indexical beliefs is assumed at the starting point. For instance, if one accepts the Lewisian view that the proper analysis of indexical beliefs requires centered propositions, then the serious challenge is to reconcile this view with the standard models of linguistic communication (cf. Weber 2103; Rudnicki 2019). 


\section{Kaplan's account of de re belief reports}

The approach to indexical beliefs I shall sketch below is inspired by the seminal analysis of de re belief reports by David Kaplan (Kaplan 1968). ${ }^{13}$ Kaplan's theory attempts to address, in the first place, the exportation problem: the issue of providing general validity criteria for inferences that start with syntactically understood de dicto belief sentences as assumptions and arrive at syntactically understood de re belief sentences as conclusions. ${ }^{14}$ For instance, everybody knows that the team that is going to beat its opponent in the next World Cup final is going to win the World Cup. This generally does not allow you to infer that there is a team of which you know that it will win the World Cup. It is clearly the latter knowledge, and definitely not the former, that may be of some interest to bookmakers. On the other hand, knowing that this team (assume that one points at a team having a training here and now) is going to win the World Cup entails that that there is a team of which you know that it is going to win the World Cup. So, it seems, in some cases believes-that or de dicto sentences enable us to derive believes-of or de re sentences. ${ }^{15}$ The exportation problem is to make explicit the additional assumptions that warrant the inference. ${ }^{16}$

Kaplan's reply to the exportation problem, as I understand it, amounts to distinguishing two kinds of de dicto belief states and two kinds of corresponding belief reports. The first category embraces beliefs that involve a special kind of representation, the second category ${ }^{17}$ does not. From the viewpoint of Kaplan's theory, we may say that de re beliefs are a special

13 Kaplan himself abandoned this view in his latter writings (cf. Kaplan 1985, 326). Although my aim here is not to provide an exegesis of Kaplan's philosophical development I shall briefly address Kaplan's reasons of dissatisfaction in the section 4.3.

14 Roughly speaking, a belief report is syntactically de dicto, if it contains a complete that-p clause, it is syntactically de re, if it contains the "believe(s) of [...] that it is F" predicate.

15 We treat knowledge as a kind of belief.

16 For a discussion of the exportation problem see (Kripke 2011).

17 Here the distinction de dicto/de re applies primarily to states and secondary to attitude reports. As such it should be distinguished from the syntactical version of the distinction. 
case of de dicto beliefs - the ones that make use of a specific class of representations. The crucial notion of representation is understood by Kaplan as a triadic relation $\boldsymbol{R}$ defined in the following manner (let $\alpha, \beta$ etc. range over vehicles of representation, whatever they $\left.\operatorname{are}^{18}\right)$ :

$\boldsymbol{R}(\alpha, x, y)(=\alpha$ represents $x$ for $y)$ iff:

(i) $\alpha$ denotes $x$,

(ii) $\alpha$ is of $x$,

(iii) $\alpha$ is vivid for $y$.

I shall call the condition (i) - the iconicity demand, the condition (ii) - the causality demand ${ }^{19}$ and the condition (iii) - the vividness demand.

I think that the best way of explaining the difference between (i) and (ii) is to consider the case through pictorial representations like photographs (Kaplan uses here the analogy with paintings). As Max Cresswell noted once:

[...] if one asks the question 'What is that a picture of?', one can be given an answer in terms of the causal ancestry of the picture. But perhaps the picture has been faked. [...] There is still a legitimate answer to the question of what a picture is the picture of. This answer can be thought of as the answer to the question of what the world would have to be like in order for things to be as the picture shows them to be. A picture of a purple cow would demand at least a world in which there was a purple cow. (Cresswell 1985, 132)

Cresswell's point is that there are in fact two kinds of representational relations: one that is causally grounded (the photograph represents someone or something that has been photographed) and the other that is founded

18 Since I do not want the described theory to be committed to any particular account of representations I am intentionally leaving the question about the nature of such vehicles open.

19 A more adequate terminology might be that of indexicality demand as it is motivated by Peircean notion of indexical sign, that is the notion of sign that is existentially (in some cases: causally) connected with its object. I decided to talk about the causality demand in order to avoid the confusion with other uses of "indexicality" in this paper. 
on the resemblance of the vehicle of representation and the thing represented (hence the iconicity). Kaplan's concept of representation presupposes the relevance of both dimensions: a vehicle of representation represents something (in Kaplan's sense) only if it resembles the thing represented and if the latter has played a special role in causing the vehicle's coming into existence. Since the relevant concepts of resemblance or similarity, according to Kaplan, apply not only to pictorial representations but also to linguistic expressions endowed with a descriptive content (like definite descriptions), that is the ones denoting objects in virtue of the fact that the objects have properties expressed in the description, resemblance must be understood here in a manner general enough to enable this. Roughly speaking, we may risk saying that Kaplan's general notion of denotation (and resemblance) amounts to presupposing that if certain facts about the denotatum had been different, the vehicle would not have been denoting this particular object. The "certain facts" in question are the ones in which the relation of generalized similarity is grounded. For instance, we might say that a road map would not have been denoting a particular road system if the number of crossroads had been different from the number of crossroad representations on the map. We might also say that, for instance, the description "the inventor of topboots' would not have been denoting Wallenstein (the actual inventor of topboots) unless Wallenstein had had been the inventor of topboots. ${ }^{20}$

The concept of vividness concerns, as Kaplan puts it, purely internal aspects of individuation:

The crucial feature of this notion is that it depends only on [...] current mental state, and ignores all links whether by resemblance or genesis with the actual world. (Kaplan 1968, 201)

20 Recently Grabarczyk (Grabarczyk 2015) introduced the idea of concepts as soft detectors used to react to undetectable properties correlated with a system's detectable internal state. It may be interesting to extend the idea of resemblance in the manner that would make it applicable to cases where a detection mechanism is involved. Here the relevant facts about possible denotata would be undetectable properties and their connections with the system's intrinsic properties. The introduced notion of resemblance is general enough to deal with such cases. 
Roughly speaking, the notion of vividness may be characterized in terms of a missing aspect that transforms iconic and causal representations of something for someone into the representations that constitute subject's acquaintance with the object. ${ }^{21}$ The idea here is that the vehicle of representation must play a sufficiently distinguished role in the subject's thoughts that intend to be about some particular object $x$. Although this general description of vividness is sufficient for purpose of the paper it should be stressed that there is currently no agreement how to describe the notion of sufficiently distinguished role. ${ }^{22}$ The important point, however, is this: each such theoretical description known to the author is consistent with the proposal introduced in the section 3 .

Equipped with iconicity, causality and vividness we are now prepared to distinguish between various cognitive situations that involve agents. Let us use asterisks (Kaplan's corner quotes) as devices that indicate that we speak about complex representational structures. Now we have a contrast between:

(4) $\exists \alpha$ [Poirot BELIEVES ${ }^{*} \alpha$ is a thief* \& $\boldsymbol{R}(\alpha$, the thief, Poirot $\left.)\right]$ and

(5) $\exists \alpha\left[\right.$ Poirot BELIEVES ${ }^{*} \alpha$ is a thief* $] \& \neg \exists \beta[\boldsymbol{R}(\beta$, the thief, Poirot) \& Poirot BELIEVES * $\beta$ is a thief*]

The first case corresponds to situations where Poirot's belief enables him to disclose who the culprit is. The second does not, on the other hand, as it fits very well to situations where Poirot thinks, for instance, that the thief is the person who broke the window but he has no idea who that person is.

21 "If the name is such, that on the assumption there exists some individual $x$ whom it both denotes and resembles, we should say that Ralph knows $x$ or is acquainted with $x$, then the name is vivid" (Kaplan 1968, 201).

22 One possible explication goes along the lines suggested by Robin Jeshion who proposes Significance Condition for singular thoughts (she uses here the terminology of mental files): "a mental file is initiated on an individual only if that individual is significant to the agent with respect to her plans, projects, affective states, motivations" (Jeshion 2010, 136). For a criticism of Jeshion account see (Geirsson 2017) where the author argues that a better alternative to Significance Condition is the condition of conscious attention. 
Roughly speaking, the exportation is warranted if (4) is true of some particular person. ${ }^{23}$

I shall argue below that the representational apparatus introduced by Kaplan can deal with de se, de hic and de nunc beliefs provided that it is treated experimentally, that is as enabling various modifications of Kaplan's original idea, modifications like relaxing conditions for (certain kinds) of representations, putting new constraints on representations, augment its formal structure etc. Such an experimental approach, I think, fits well with Kaplan's declarations (cf. Kaplan 1968, 204).

\section{Extending Kaplan's account}

To start, I shall consider three modifications of Kaplan's representational approach.

The first one is that of enriched relativization. It seems that particular acts of representing involve a setting or a situation in which the representation takes place. This setting or situation, just like all the others, comprises a potentially infinite number of aspects or properties. Some of these aspects are relevant for the particular act of representing, while others are not. I would like to suggest that de se, de hic and de nunc beliefs are ones that engage particular kinds of such aspects: the agent that plays a particular role in the situation, its time and its location. In fact, the agent has already explicitly been featured as an argument of the representational relation - the idea, in a sense, is just to generalize this relativization into other aspects.

The second adjustment is that of situation dependence. Mere relativization (even enriched) does not mean much and, in particular, it does not entail that there exists any sort of dependence between the relevant aspect of the situation and the object of the representation. However, we may independently consider the possibility of such a dependence. In general, we may single out a class of situations where the object of the representation

23 Due to space limitations, over the course of presentation I am ignoring several features of Kaplan's approach like the issue of the relative and gradable nature of vividness, the role of type-token distinction etc. 
is a function of this or another aspect of the setting. If $c$ is such a circumstance, setting or situation we may replace our original relation $\boldsymbol{R}(\alpha, x, y)$ with $\boldsymbol{R}(\alpha, x, y, c)$ (meaning: $\alpha$ is a representation of $x$ for $y$ at $c$ ) and consider the class of cases for which $\boldsymbol{R}(\alpha, f(c), y, c)$. In the cases we are interested in: $c=\langle a, t, l\rangle$, where $a$ is the agent, $t$ is the time and $l$ is the place or location and the functional dependence is therefore of the simplest kind, as the object represented is identical with one of the aspects of situation (that is either with the agent $a$ of the situation, the time $t$ or the location l). Hence we may consider three general reflexive cases: the first in which we deal with a representation of an agent for the agent (represented as $\boldsymbol{R}(\alpha, a, a,\langle a, t, l\rangle))$, the second where we deal with a representation of a time or instant at the time or instant (represented as $\boldsymbol{R}(\alpha, t, a,\langle a, t, l\rangle))$ and the third in which we deal with a representation of a location or place at the location or place (represented as $\boldsymbol{R}(\alpha, l, a,\langle a, t, l\rangle)$ ).

The third idea is that of uniqueness. Some representations may be unique in the following sense: the change in the relevant aspect of the situation or setting requires a change of the vehicle of representation. Uniqueness basically means that a representation cannot occur if the circumstances are not of the appropriate kind or, alternatively, that it is unavailable for subjects who are not in the situation of the required kind. Due to that we may call such representations elusive. The concept of situations' relevant aspect differs for distinct kinds of indexical beliefs. Roughly speaking, in the case of de se attitudes it is the agent of the situation, in the case of de nunc attitudes it is the time of the situation, in the case of de hic attitudes it is the location of the situation.

The concept of uniqueness may sound familiar to those who endorse the idea of hybrid names (cf. Künne 1992; Textor 2007; Textor 2015). Hybrid names are names consisting of linguistic expressions and circumstances in which linguistic expressions occur. Although hybrid name theorists differ with respect to the question about the exact role of circumstances in reference fixing, they all agree that they are partially individuated by the circumstances in question. In the case of hybrid names, one is entitled to say that "[...] only utterances of 'I' by the same person, and only simultaneous utterances of 'the present moment' are occurrences of the same hybrid proper name" (Künne 1992, 725). Parallel considerations apply to unique 
representations: de se representations may re-occur (that is they are occurrences of the same representation) only if the agent remains fixed, de nunc representations may re-occur only if the time remains fixed, de hic representations may re-occur only if the location remains fixed. However, there are also important differences between hybrid names and unique representations. These differences do not show that the two concepts cannot be combined within a single theory of representations or hybridity, they show rather that combining them would require substantial enrichment of one concept with the features of the other. Firstly, unique representations do not have to be linguistic or even partially linguistic. ${ }^{24}$ They have to be mental representations, whatever the latter are. Hybrid proper names, on the other hand, essentially contain linguistic and circumstantial components. Secondly, unique representations of a particular kind (de se, de nunc or de hic) are representations that are iconic, causal and vivid. Nothing in the idea of hybrid name presupposes the three aspects. Thirdly, hybrid names are mereological sums of two kinds of components that both have properties determinative for the reference of a hybrid name. The first, the linguistic component, is endowed with meaning that tells how circumstances relate to the referent of the hybrid name, the second, the circumstantial component, saturates the meaning and gives reference. In contrast to hybrid names, unique representations do not have a special mereology: they may possibly be complex but have no referentially relevant parts or components. Fourthly, hybrid names as containing circumstances as parts are intrinsically functional upon situations.

Another theory that embraces the concept of uniqueness has been proposed by Peacocke (1981) and developed later by Higginbotham (Higginbotham 2003), see (Capone 2016, 232-36). Peacocke introduced the idea of special modes of presentations $\left(\operatorname{self}_{x}\right)$ and $\left(\right.$ now $\left._{t}\right)$ that might be, respectively, solely constituents of the thoughts of a person $x$ when $x$ is thinking about himself or herself and constituents of the thoughts entertained at the time $t$ that concern the time $t$. Higginbotham develops a view according to which sentences that have a de se interpretation employ $\left(\operatorname{self}_{x}\right)$ at the level

24 On the other hand, they have to be semiotic in the full-blooded sense (cf. Konderak 2017). 
of logical form. ${ }^{25}$ The difference between the view defended in this paper and the views of Peacocke and Higginbotham is, firstly, that the former makes no use of the concept of mode of presentations and makes no use of the notion of sense (or its Carnapian counterpart-intension). As I shall explain below at the level of semantic interpretation belief reports state that there is a relation between an agent and a mental propositional representation, not between an agent and a proposition or a Fregean thought. Secondly, Peacocke-Higginbotham's idea of de se mode of presentation is not committed to the special epistemology of representation developed in this paper. The two differences, however, should not obscure the similarities between the approaches. One might, for instance, treat the account sketched in this paper as psychologized version of Fregean account as well as consider supplementing the Peacocke-Higginbotham's views with the special epistemology of representation. This will, actually, meet Peacocke's demand that one of the crucial tasks of the philosophy of mind is to say what the capability of thinking of objects under particular modes of presentations amounts to (Peacocke 1981, 194).

We are now in a position to define a minimal generic notion of indexical representation ("minimal" because it comprises just three aspects of the situation, "generic" because it uses the general concept of appropriateness that becomes definite for particular sorts of indexical beliefs (see below)):

For all $\alpha, x, y$ and $c=\langle a, t, l\rangle$ (for some agent $a$, time $t$ and location l):

$\boldsymbol{R} \boldsymbol{I}(\alpha, x, y, c)(=\alpha$ indexically represents $x$ for $y$ in a situation $c)$ iff

1. $\boldsymbol{R}(\alpha, x, y, c)(=\alpha$ represents [in the sense of Kaplan] $x$ for $y$ in a situation $c)$

2. $x=f(c)$ (situation dependence)

3. for all $c^{\prime}$ : if $c$ is appropriately different from $c^{\prime}$, then it is necessary that: $\neg \boldsymbol{R}\left(\alpha, x, y, c^{\prime}\right)$ (uniqueness)

25 Higginbotham stresses that there are differences between his and Peacocke but since they both embrace uniqueness of de se mode of presentation they are of secondary importance for this paper. 
The concept of being appropriately different is intended to capture the fact that the indexical nature of representation does not mean a sensitivity to the change of the situation as a whole but rather a sensitivity to the change in one of its aspects (its agent, its time or its location). Two situations may be different but, if the difference is not the effect of the change in the appropriate aspect of the situation, the vehicle of representation might remain unchanged (an agent might clearly have constant de se representation of himself or herself at different times or at different locations).

I assume here a notational distinction between the subject of representation and the agent of the situation but one may, for the sake of notational simplicity, reduce $\boldsymbol{R}(\alpha, x, y,\langle a, t, l\rangle)$ to $\boldsymbol{R}(\alpha, x,\langle a, t, l\rangle)$ if she keeps in mind the assumption that the subject that represents and the agent of $c$ are, in fact, identical. I will follow this convention below but the reader is kindly asked to bear in mind that the subject of the representation is always indirectly represented in $\boldsymbol{R}(\alpha, x,\langle a, t, l\rangle)$.

Corresponding notions of de se, de hic and de nunc representations may be defined as follows:

(Representing de se)

For all $\alpha, x, a, t, l$ :

$\boldsymbol{R} \boldsymbol{S}(\alpha, x,\langle a, t, l\rangle)(=\alpha$ is a de se representation of $x$ at the situation involving the agent $a$, the time $t$ and the location $l$ iff

1. $\boldsymbol{R}(\alpha, x,\langle a, t, l\rangle)(=\alpha$ represents $x$ at the situation involving the agent $a$, the time $t$ and the location $l$ )

2. $x=a$

3. for all $a^{\prime}$ : if $a^{\prime}$ is different from $a$, then - for each $t^{*}$ and $l^{*}$-it is necessary that: $\neg \boldsymbol{R}\left(\alpha, x,\left\langle a^{\prime}, t^{*}, l^{*}\right\rangle\right)$

(Representing de hic)

For all $\alpha, x, a, t, l$ :

$\boldsymbol{R H}(\alpha, x,\langle a, t, l\rangle)(=\alpha$ is a de hic representation of $x$ at the situation involving the agent $a$, the time $t$ and the location $l$ ) iff

1. $\boldsymbol{R}(\alpha, x,\langle a, t, l\rangle)(=\alpha$ represents $x$ at the situation involving the agent $a$, the time $t$ and the location $l$ )

2. $x=l$

3. for all $l^{\prime}$ : if $l^{\prime}$ is different from $l$, then-for each $a^{*}$ and $t^{*}$-it is necessary that: $\neg \boldsymbol{R}\left(\alpha, x,\left\langle a^{*}, t^{*}, l^{\prime}\right\rangle\right)$ 
(Representing de nunc)

For all $\alpha, x, a, t, l$ :

$\boldsymbol{R} \boldsymbol{N}(\alpha, x,\langle a, t, l\rangle)(=\alpha$ is a de nunc representation of $x$ at the situation involving the agent $a$, the time $t$ and the location $l$ ) iff

1. $\boldsymbol{R}(\alpha, x,\langle a, t, l\rangle)(=\alpha$ represents $x$ at the situation involving the agent $a$, the time $t$ and the location $l$ )

2. $x=t$

3. for all $t^{\prime}$ : if $t^{\prime}$ is different from $t$, then-for each $a^{*}$ and $l^{*}$-it is necessary that: $\neg \boldsymbol{R}\left(\alpha, x,\left\langle a^{*}, t^{\prime}, l^{*}\right\rangle\right)^{26}$

$\boldsymbol{R I}, \boldsymbol{R} \boldsymbol{S}, \boldsymbol{R H}$ and $\boldsymbol{R} \boldsymbol{N}$, recall, are all defined in terms of the original Kaplanian relation of representation. Hence, they all meet iconicity, causality and vividness demands.

Now, just like de re belief reports can be analyzed along the lines depicted in (4) the reports similar to (2) (A believes that she herself is $F$ ) receive the following representation:

(6) $\exists \alpha\left[\right.$ A BELIEVES $* \alpha$ is $\left.\mathrm{F}^{*} \& \boldsymbol{R} \boldsymbol{S}(\alpha, \mathrm{A},\langle\mathrm{A}, \mathbf{t}, \mathbf{l}\rangle)\right]$

and the reports like (3) (A believes that she (but not that she herself) is F) the following:

(7) $\exists \alpha\left[\right.$ A BELIEVES $* \alpha$ is $\mathrm{F}^{*} \& \neg \boldsymbol{R} \boldsymbol{S}(\alpha, \mathrm{A},\langle\mathrm{A}, \mathbf{t}, \mathbf{l}\rangle)^{27}$

Due to the risk of boring my readers I will not go through the analogous cases of de hic and de nunc beliefs. Note that (7) can be made true by the entire class of different states of affairs that involve the psychological state of the agent A. Firstly, (7) is true if any of the three constitutive conditions for $\boldsymbol{R} \boldsymbol{S}$ fails to obtain. This might happen, for instance, if $\alpha$ is not vivid, it may happen if it is not iconic or if it does not meet the causality demand. This embraces five possible kinds of cases if we assume that vividness requires iconicity and causality and even more if we deny that assumption.

26 Points 1 and 2 of each definition may just be, of course, reduced to overtly reflexive $\boldsymbol{R}(\alpha, a,\langle a, t, l\rangle), \boldsymbol{R}(\alpha, t,\langle a, t, l\rangle)$ and $\boldsymbol{R}(\alpha, l,\langle a, t, l\rangle)$ respectively.

${ }_{27}$ In cases where the agent has no disposition at all to regard himself or herself as being $\mathrm{F}$ (she suffers from crime related amnesia, for instance) the reading may be even strengthen to: $\exists \alpha$ [A BELIEVES ${ }^{*} \alpha$ is $\mathrm{F}^{*} \& \forall \beta$ (A BELIEVES $* \beta$ is $\mathrm{F}^{*} \Rightarrow$ $\neg \boldsymbol{R} \boldsymbol{S}(\beta, \mathrm{A},\langle\mathrm{A}, \mathbf{t}, \mathbf{l}\rangle)]$ which entails $(7)$. 
Combining this with other cases where situation dependence or uniqueness fails we arrive at twenty-three kinds of situations that can make (7) true. This illustrates the fact that there is no simple way of connecting belief reports (at least some belief reports) and kinds of belief states that fit the belief reports in question. However, at the very same time the semantics of belief reports indicates features that every belief state must have in order to qualify as a potential truthmaker of the belief report.

The following bigger picture emerges from the analysis just presented. Beliefs, roughly, may be described as relations between agents and propositional representational structures. If we treat as criterial (for being a de dicto propositional attitude) the fact that the relation takes a propositional structure as an argument, then all the kinds of beliefs described above count as de dicto. De re beliefs, from that perspective, are the ones that involve relation to the propositional structure that contains at least one iconic, causal and vivid representation. Indexical beliefs are, consequently, the ones that involve the relation to the propositional structure containing iconic, causal and vivid representation that is, additionally, relativized, situation dependent and unique. In the case of de se beliefs this commits us to the well-known Fregean self-presentation demand as our definition of de se entails that "everyone is presented to himself in a special and primitive way, in which he is presented to no one else" (Frege 1956).

The theory sketched above differs in several aspects from the most popular approaches to indexical beliefs. Firstly, the approach is not committed in any way to special kinds of de se, de hic or de nunc or relativized contents (it is, however, committed to representations of limited accessibility, more about that below). In particular, it does not describe beliefs in the Lewisian manner, that is as relations between agents and properties. It also does not make use of centered worlds (cf. Stalnaker 2008) as it postulates a more fine-gained notion of belief described in terms of representations. Secondly, it differs also from the approach of John Perry as it does not introduce the special concept of a belief state that determines the proposition relatively to a situation. Here, however, the situation looks slightly different as the discussed notion of representation is clearly situation relative. If one thinks, therefore, that using a particular representation shapes the belief state of an agent, then the present account may be seen as a representationalist variant of Perry's approach. 
The account presented offers a solution to all three problems of locating beliefs described by Feit and Capone. Firstly, de se beliefs differ from nonde-se beliefs in the kind of representation involved. In particular, akin de re representations are neither situation dependent nor unique. Secondly, the semantic problem is solved in two steps. One is by noting that there is no need to postulate a special semantic value of "I" or "she herself" other than the referent. Another is by noting that at the level of semantic representation the belief reports that contain such phrases explicitly make use of a special relation of representation. Thirdly, if we interpret the Geach problem as a question regarding the interpretation of our propensity to treat as cases of samebelieving situations, for example like the one where both Donald and Hilary think that they will win the next presidential elections, ${ }^{28}$ then the corresponding belief reports are:

(8) $\exists \alpha$ [Hilary BELIEVES $* \alpha$ will win* \& $\boldsymbol{R} \boldsymbol{S}(\alpha$, Hilary, $\langle$ Hilary, $\mathbf{t}, \mathbf{l}\rangle))$

(9) $\exists \alpha$ [Donald BELIEVES * $\alpha$ will win* \& $\boldsymbol{R} \boldsymbol{S}(\alpha$, Donald, $\langle$ Donald, $\mathbf{t}, \mathbf{l}\rangle))$

It is, I think, clearly visible that both Hilary and Donald are related to representations that have not only isomorphic structure but also involve a single kind of representation. This, I think, suffices for explaining the samebeliving intuition in question. On the other hand, due to the uniqueness demand, they cannot be related to the same representation of the relevant kind. This naturally explains the opposite intuition.

\section{Loosening the requirements put on indexical beliefs: five doubts}

I conclude that the theory sketched above offers a promising analysis of some aspects of the problem of indexical belief. My plan now is to discuss some potential challenges that the theory must face.

28 We must, of course, keep in mind that we also have an opposite propensity to treat such cases as the ones of non-samebelieving. 


\subsection{De re beliefs about oneself}

John Perry, after discussing the proposals of Kaplan and Hintikka construing de re attitudes as special cases of de dicto attitudes, defied the idea of the applicability of such an approach to indexical beliefs on the following grounds:

However well these proposals deal with other phenomena connected with de re belief, they cannot help with the problem of the essential indexical. They tighten the requirements laid down by the original proposal, but those were apparently already too restrictive. If in order to believe that I am making a mess I need not have any conceptual ingredient $\alpha$ that fits me, a fortiori I am not required to have one that is a vivid name of myself for me, or one that picks out the same individual in every possible world compatible with what I believe. (Perry 1979, 11)

Immediately Perry also provided the examples of de re beliefs of an agent about oneself that are not indexical (Perry 1979, 12). One goes as follows. Imagine that I see the reflection of the messy shopper in the mirror and I express my belief by saying "He is making a mess." By doing this I express a de re belief about myself. However, if I am not aware that this is my reflection I see, this de re belief is not a de se one. ${ }^{29}$

I have no doubts that such examples conclusively prove that the class of de se beliefs and the class of de re beliefs about oneself are distinct. Does this fact pose an insuperable challenge to the theory sketched above?

There can be little doubt that in such cases the demands of iconicity, causality, vividness and enriched relativization are met. What about situation dependence and uniqueness? If we equate the situation, as we did, with the triumvirate that consists of an agent, a location and an instant, then the situation dependence means that the representation involved in de se cases is a function of the agent of the situation. And this is clearly the case: accidentally or not, the object represented is identical with the agent that has the relevant belief. However, the uniqueness demand is not met by the

29 Similar cases have been considered in the early 70's by Ernest Sosa (Sosa 1970, 893). Perry refers to such cases as cases of "belief of the person one happens to be" (Perry 1998), Recanati (Recanati 2007) calls such beliefs "accidental de se." 
agents involved in the cases of accidental de se beliefs. Other person may also see the reflection of the messy shopper and utter "He is making a mess." I see no reason to claim that there is a difference in the kind of vehicle of representation between myself (having accidental de se belief) and such persons (having a regular de re belief about myself). Therefore, the presented account of de se beliefs is not undermined by the discussed examples.

Inspired by scenarios involving accidental de se beliefs Boër and Lycan (Boër and Lycan 1980) suggested that there is no real contrast between the case of indexical beliefs and the case of non-indexical de re beliefs. The reason for this is that one might consider scenarios involving non-indexical de re beliefs that strictly follow the structure of cases involving accidental de se attitudes. One can, for instance (cf. Boër and Lycan 1980, 448-50), consider the story of John who sees two distant persons. One is followed by a predator while the other looks perfectly safe. John dubs the first person 'Van' and the other 'Wilfrid.' In fact, Van is just a mirror reflection of Wilfrid and the mirror reflection reveals the predator that cannot be seen in the flesh. It seems that we have here two de re beliefs about single person that correspond to accidental and regular de se beliefs.

The framework sketched above, I believe, enables to see the difference between the two cases. If Van-Wilfrid scenario involves unique representations, then both cases can only be perceptually unique (Van is the men that looks thusly from here now while Wilfrid is the men that looks differently from here now). Therefore, there is a symmetry between perceiving Van and perceiving Wilfrid as the change in the relevant aspect of the perceptual situation enforces the change in the representation itself (the change of the Van-like representation into the Wilfrid-like representation, for instance). However, as it has been noted above, no parallel symmetry in the cases of regular and accidental de se exists as accidental de se cases do not meet the uniqueness requirement with respect the relevant aspect of the situation.

\subsection{Selfless knowledge}

A different challenge is, I think, posed by the eventuality that "in order to believe that I am making a mess I need not have any conceptual ingredient $\alpha$ that fits me." The idea fits well with the cluster of theories developed in other papers of John Perry (cf. Perry 1986; and Kim 2010, 60-62) 
where the ideas of thought without representation and the essentially selfless knowledge are introduced and discussed. Firstly, Perry argues there that it is, in principle, possible to have thoughts about something without having a representation of that thing. Secondly, he notes that many thoughts that play a motivational role in causing our actions seem to be selfless. Now, if we enable thoughts without representation and the idea of intrinsically selfless knowledge, then the possibility that at least some de se thoughts involve no representation of the subject may look compelling. ${ }^{30}$ And if it is compelling - one may suggest - an adequate account of de se beliefs cannot appeal to representations regardless of their elusiveness.

We have, therefore, two competing pictures of locating beliefs: one committed to elusive representations and another committed to non-self-representationalism. I think that the phenomenon of essential indexicality together with other broadly accepted observations regarding the explanation of human actions does, in general, favor the representationalism defended in this paper. Suppose that I am just about to write down the word "self." In order to do that I have to press a certain sequence of keys on the keyboard in front of me. The keys are thusly located with respect to me, the sequence of little key-pressing actions I have to perform is determined partially by the goal I have. All of these shape the action I actually perform. The objects involved (the keyboard, keys and the sequence of letters qua the intended result) might be conceived as entities that play agent-relative roles (cf. Perry 2000, 326). I clearly may have knowledge of all such things without representing them as accordingly related to me. However, can such knowledge be solely responsible for the explanation of action in cases where indexical beliefs seem necessary? Consider our absent-minded referee again. It is an essential feature of Monica's belief that by gaining the knowledge about the authorship of the paper she is gaining the knowledge that is $e x$ plicitly about her. This is why we are in the position to dismiss all corresponding non-egocentric explanations that are not accompanied by the additional belief that she herself plays a corresponding non-egocentric role. This is what makes her belief essentially indexical. Hence, there are reasons

30 Naturally, some de se thoughts may engage the representation of the subject but the very fact that the opposite is possible entails that containing a representation (of any sort) is not obligatory for being a de se thought. 
to think that the classes of selfless thoughts and indexical thoughts are disjoint. Due to this fact one cannot argue against elusive representations on the basis of the possibility of thoughts without self-representation.

\subsection{En report requirement}

I should like now to consider a different kind of worry. The approach presented above is based on David Kaplan's ideas and in his later writings Kaplan himself expressed skepticism regarding this stage of his philosophical development (cf. Kaplan 1985, 326). He also suggested that his content/character distinction constitutes a more promising framework for dealing with motivational role of beliefs. Kaplan's skepticism concerns primarily his conception of de re propositional attitudes and, as such, questions the bigger picture of beliefs described at the end of the section three. A natural reaction to Kaplan's skepticism is noting that even though the bigger picture may be incorrect, this does not prove that the en report requirement (which consists in the demands of iconicity, causality and vividness) is not necessary for indexical beliefs. In fact, it is difficult to imagine the cases in which one has a locating belief without being en report with its object. So, even if Kaplan is right about some de re cases, his observation does not extend to de se, de hic and de nunc ones.

An alternative reply may stress a difference between expressing a singular proposition and believing (and eventually grasping) it. It is, I think, by no means accidental that Kaplan's example concerns assertion (not belief). Asserting something undoubtedly involves a public language with all its reference-fixing aspects and ubiquitous linguistic conformism. Gareth Evans noted once that expressions are often used deferentially, that is with the intention of conforming to the use of others (Evans 1973, 205). In such cases a person may clearly express a proposition even though her beliefs may gave her an inadequate idea about the object she is referring to and, consequently, about the proposition she actually expresses. This, among other things, enables her to express a proposition without entertaining it. The first child example used by Kaplan (Dthat ('the first child to be born in the twenty-first century') will be bald) might be interpreted as an interesting case of a similar type: we expressed a singular proposition, which we were not yet capable of grasping (in 1978 when the example was given). 
The difference between Evans deferential uses and the Kaplan example would be that in the latter case we conform to the use of a description in the whole linguistic community while instrumentally employing this usage as a demonstrating procedure. Nothing similar applies to deferential uses. Despite this difference, however, both cases may potentially lead to the assertion of the proposition that $p$ without belief that $p$.

I do not want to favor one of the two possible replies just presented. The important point is this: Kaplan's worry regarding his own theory does not automatically extend to self-locating beliefs, besides any such extension is highly problematic.

\subsection{Propositions of limited accessibility}

We have seen that one of the most important features of the described approach to self-locating beliefs is the fact that it postulates a class of unique representations - ones that are not repeatable if the change in the situation affects it in a particular manner. This may suggest that our theory is committed to propositions of limited accessibility (PLA).

The idea of propositions of limited accessibility was introduced by John Perry who described it as claiming that:

[...] there is a class of propositions which can only be expressed in special circumstances. In particular, only I could express the proposition I expressed when I said 'I am making a mess.' Others can see, perhaps by analogy with their own case, that there is a proposition that I express, but it is in a sense inaccessible to them. (Perry 1979, 16)

Although Perry described theories that are committed to PLA as offering a consistent solution to the problem of essential indexicality, he expressed his skepticism towards them. Firstly, he pointed out that one needs additional metaphysical reasons to enable PLA. Secondly, he suggested that such reasons must somehow presuppose the ontology of „private perspectives” (Perry 1979, 16). This, in turn, questions our belief in „the common actual world" (Perry 1979, 16).

It is, therefore, important to stress that the theory sketched in this paper is literally committed not to propositions of limited accessibility but rather 
to representations of limited accessibility (RLA). Now, we clearly do not have to equate PLA and RLA. Here are examples of three theories that are may embrace the distinction. Firstly, the representational theory of the mind, for instance, offers a theoretical framework that describes attitudes as directly related to mental representations and indirectly (via mental representations) to contents (including the propositional ones). Any account that sympathizes with the representational theory of the mind might, therefore, postulate RLA but deny any sort of commitment to PLA. Secondly, similar remarks apply to theories that approach the concept of proposition in a classificatory way. ${ }^{31}$ Thirdly, despite all the differences between hybrid names and unique representations there is one important parallel between the two ideas. Both unique representation and hybrid names are not contents but rather means of expression of the content. By distinguishing between propositions and representations the theory sketched in this paper assumes that objects of attitudes (representations) and semantic values of sentences (propositions) are different entities.

Since Stoic times there have been two main traditions of interpreting the concept of proposition. The first, represented for instance by Bolzano and Frege, construed them as abstract, mind independent entities. The second, represented for instance by medieval nominalists, construed them as mind dependent entities, often expressions of the mental language. Neither of the two theories, I think, would be happy to accept propositions of limited accessibility and neither would have a problem with representations of limited accessibility. The only theory that may be somehow committed to PLA is the one that equates propositions with representations and, at the very same time, treats them as abstract or, at least, completely mind independent entities (like states of affairs or situations). Such a theory would endow cognitive subjects with the capacity of having thoughts or attitudes that are direct relations to such mind independent entities. If we interpret

31 Here John Perry stresses the importance of the representation-proposition distinction: "My conception [...] is that propositions are abstract objects we use to classify cognitive states, especially states that involve representations. Propositions are not representations, but abstract objects that we use to classify states and events by the requirements of their truth (or some other form of success imposed on the rest of the world)" (Perry 2012, 27). 
the theory presented in this paper along such lines, then it is unquestionably committed to representations qua propositions of limited accessibility, However, I see no rationale for interpreting or developing it in that manner.

\subsection{The account is "too fine-grained"}

One might worry that the view defended in this paper provides too finegrained criteria of belief individuation. The uniqueness constraint prohibits that indexical beliefs can be entertained in situations that appropriately differ from the ones where they are actually entertained. However, it seems that there is a sense in which my thought that I have two hands now (entertained at the time t) and my thought that I have two hands now (entertained at the time $t^{\prime}$ different from $t$ ) have the same content. ${ }^{32}$ One aspect of the problem has already been addressed above: the case of two nowthoughts seems to be a temporal counterpart of the example used to illustrate Geach problem. The analysis of the cases, therefore, is similar: on the one hand, we may explain the intuition of sameness of beliefs by the fact that the two beliefs are isomorphic. There is, however, another aspect of the problem that deserves attention. If we follow strictly the observation that our theory treats beliefs as relations to mental representations, then the following possibility emerges: distinct mental representations might be linked to a single propositional content. If we embrace this possibility, there are at least two levels at which beliefs might be individuated. One is the level of propositional representations. At this level, two de nunc thoughts

32 The anonymous referee asked me to consider examples of two first person thoughts that I have hair today entertained on two consecutive days. Since my account says nothing about hoc die attitudes I decided to change the example to two de nunc thoughts. However, the point raised by the referee motivates another important question: should the account sketched in this paper be generalized to other sorts of indexical thoughts (today-thoughts, yesterday-thoughts, there-thoughts, you-thoughts etc.). My initial reaction is that is should not as the thoughts in question seem not to require uniqueness (I agree here with Frege's famous observations concerning "today" and "yesterday," I denied this generalization for demonstrative also when discussing the case of accidental de se). However, I do not have at the present moment the general criterion of demarcation and I would like to stress that this important issue requires further studies. 
entertained at different times might be similar but never strictly type-identical. Another is the level of propositional content. Here, despite being related to two distinct propositional representations, two de nunc thoughts entertained at different times might have the same propositional content. We have to be very careful here, however, when it comes to the analysis of particular examples. On the one hand, we might follow Prior's intuitions (Prior 1970) that redundancy theory of the present (or redundancy theory of being here) correctly captures the content of now-thoughts and herethoughts so my thought that I have two hands now is nothing more than the thought that I am having two hands. On the other hand, a similar approach to de se thoughts seems superficial and controversial. ${ }^{33}$ That having been said, at the present moment I prefer to treat this application of the propositional content/proportional representation distinction as the signpost of one of several ways in which the theory described in this paper might be developed.

\section{Conclusions}

I have argued that a modification of Kaplan's approach to de re belief reports can be consistently extended to cover various cases of indexical beliefs. The resulting theory postulates a special class of unique circumstancedependent representations. However, it is neither committed to propositions of limited accessibility, nor to any sort of special indexical contents. The theory has numerous merits. Firstly, it offers a solution to psychological and semantic problems of indexical beliefs. Secondly, it enables us to solve the Geach problem. Thirdly, it has the resources to distinguish cases of regular de se and accidental de se beliefs as well as to address Boër and Lycan worries that there is no important difference between indexical and non-indexical de re attitudes. Fourthly, it gives a general and consistent picture of de dicto, de re and indexical attitudes. In particular, it indicates the similarities and dissimilarities between the three classes of attitudes. Last but not least, it seems to promise an area for further interesting

33 Nevertheless, it still might be developed in the framework of egocentric logic developed by Prior (cf. Prior 1968, 135-44). 
philosophical developments. The account seems, for instance, to fit very well to the representational theory of the mind, the theories that appeal to the idea of representations having forms (like the mental orthography approaches), it may also be perceived as supporting the view of concepts as mental representations (cf. Margolis and Laurence 2007). All these merits, I think, support the theory as being worthy of further investigation and development.

\section{Acknowledgements}

Ideas presented in this paper were discussed at Semantic Content and Conversational Dynamics workshop (Barcelona), Philang 2017 (Lodz), South American Congress of Analytic Philosophy (Campinas), European Congress of Analytic Philosophy (Munich), during my short visit at the University of Konstanz and at the meeting of Institute of Philosophy of the Czech Academy of Sciences. I would like to thank the audiences and the anonymous reviewers of Organon $F$ for insightful comments and suggestions.

\section{Funding}

The work on this paper was funded by National Science Center, Poland, grant under award number 2013/11/B/HS1/03947.

\section{References}

Boër, Steven E., and Lycan, William G. 1980. "Who, me?" The Philosophical Review LXXXIX (3): 427-66. https://doi.org/10.2307/2184397

Capone, Alessandro. 2016. The Pragmatics of Indirect Reports. Socio-Philosophical

Considerations. Dordrecht: Springer. https://doi.org/10.1007/978-3-319-41078-4 Cappelen, Herman, and Dever, Josh. 2013. The Inessential Indexical: On the Philosophical Insignificance of Perspective and the First Person. Oxford: Oxford University Press. https://doi.org/10.1093/acprof:oso/9780199686742.001.0001

Castañeda, Hector N. 1966. "He': A Study in the Logic of Self Consciousness." Ratio 7: 130-57.

Chierchia, Gennaro. 1989. "Anaphora and Attitudes De Se." In Semantics and Contextual Expressions, edited by Renate Bartsch, Johan van Benthem, and Peter van Emde Boas, 1-31. Dordrecht: Foris.

Ciecierski, Tadeusz. 2016. "Linguistic Criteria of Intentionality." Studies in Logic, Grammar and Rhetoric 46 (1): 35-58. https://doi.org/10.1515/slgr-2016-0032 
Corazza, Eros. 2004. Reflecting the Mind. Oxford: Oxford University Press. https://doi.org/10.1093/019927018X.001.0001

Cresswell, Max. 1985. Structured Meanings. Cambridge (MA): The MIT Press.

Evans, Gareth. 1973. "The Causal Theory of Names." Proceedings of the Aristotelian Society, Supplementary Volumes 47: 187-225. https://doi.org/10.1093/aristoteliansupp/47.1.187

Feit, Neil, and Capone, Alessandro. 2013. "The Problem of De Se Attitudes." In Attitudes De Se. Linguistics, Epistemology, Metaphysics, edited by Neil Feit and Alessandro Capone, 1-25. Stanford: CSLI Publications.

Frege, Gottlob. 1956. "The Thought: A Logical Inquiry." Mind 65: 289-311.

Geirsson, Heimir. 2017. "Singular Thought, Cognitivism, and Conscious Attention." Erkenntnis 83 (3): 1-14. https://doi.org/10.1007/s10670-017-9905-3

Gillon, Brendan S. 1990. "Ambiguity, Generality, and Indeterminacy: Tests and Definitions." Synthese 85: 391-416. https://doi.org/10.1007/BF00484835

Grabarczyk, Paweł. 2016. Concepts as Soft Detectors. New Ideas in Psychology 40: 86-93. https://doi.org/10.1016/j.newideapsych.2015.03.001

Higginbotham, James. 2003. "Remembering, Imagining, and the First Person." In Epistemology of Language, edited by Alex Barber, 496-535. Oxford: Clarendon. https://doi.org/10.1093/acprof:oso/9780199239313.003.0012

Jaszczolt, Kasia. 2013. "Contextualism and Minimalism on De Se Belief Ascription." In Attitudes De Se. Linguistics, Epistemology, Metaphysics, edited by Neil Feit and Alessandro Capone, 69-103. Stanford: CSLI Publications.

Jeshion, Robin. 2010. "Singular Thought: Acquaintance, Semantic Instrumentalism and Cognitivism." In New Essays on Singular Thought, edited by Robin Jeshion, 105-40. Oxford: Oxford University Press. https://doi.org/10.1093/acprof:oso/9780199567881.003.0005

Kaplan, David. 1968. "Quantifying in." Synthese 19 (1-2): 178-214. https://doi.org/10.1007/BF00568057

Kaplan, David. 1985. "Dthat." In The Philosophy of Language, edited by Aloysius Martinich, 315-28. Oxford: Oxford University Press.

Kim, Hanseung. 2010. "Two Notions of Indexicality." Semiotica 180 (1): 47-67. https://doi.org/10.1515/semi.2010.030

Konderak, Piotr. 2017. "The Conscious Semiotic Mind." Studia Semiotyczne 31 (1): 67-89. https://doi.org/10.26333/sts.xxxi1.05

Kripke, Saul. 2011. "Unrestricted Exportation and Some Morals for the Philosophy of Language." In Philosophical Troubles, 322-50. Oxford: Oxford University Press. https://doi.org/10.1093/acprof:oso/9780199730155.001.0001

Künne, Wolfgang. 1992. "Hybrid Proper Names." Mind 101 (404): 721-30. https://doi.org/10.1093/mind/101.404.721 
Lewis, David. 1979. "Attitudes De Dicto and De Se." Philosophical Review 88 (4): 513-43.

Margolis, Eric, and Laurence, Stephen. 2007. "The Ontology of Concepts-Abstract Objects or Mental Representations?" Nô̂s 41 (4): 561-93. https://doi.org/10.1111/j.1468-0068.2007.00663.x

Peacocke, Christopher. 1981. "Demonstrative Thought and Psychological Explanation." Synthese 49 (2): 187-217. https://doi.org/10.1007/BF01064298

Perry, John. 1979. "The Problem of the Essential Indexical." Nô̂s 13 (1): 3-21.

Perry, John. 1986. "Thought without Representation." Proceedings of the Aristotelian Society, Supplementary Volumes 60: 137-66. https://doi.org/10.1093/aristoteliansupp/60.1.137

Perry, John. 1998. "Myself and I." In Philosophie in Synthetischer Absicht, edited by Marcelo Stamm, 83-103, Klett-Cotta. Reprinted in (Perry 2000, 325-39).

Perry, John. 2000. The Problem of Essential Indexical and Other Essays. Stanford: CSLI Publications.

Perry, John. 2013. "Self-Locating Beliefs." In Attitudes De Se. Linguistics, Epistemology, Metaphysics, edited by Neil Feit and Alessandro Capone, 385-408. Stanford: CSLI Publications.

Peterson, Philip L. 1997. Fact, Proposition, Event. Dordrecht: Kluwer Academic Publishers. https://doi.org/10.1007/978-94-015-8959-8

Prior, Arthur N. 1968. Papers on Time and Tense. Oxford: Oxford University Press.

Prior, Arthur N. 1970. "The Notion of the Present." Studium Generale 23: 245-48.

Recanati, François. 2007. "Imagining De Se." https://halshs.archives-ouvertes.fr/file/index/docid/160757/filename/de_se_imagining11.pdf

Rudnicki, Jakub. 2019. "Centred Propositions, what is Asserted, and Communication." Theoria. https://doi.org/10.1111/theo.12215

Schlenker, Philippe. 2011. "Indexicality and De Se Reports." In Semantics. An International Handbook of Natural Language Meaning. edited by Klaus von Heusinger, Claudia Maienborn, and Paul Portner, 1561-1604. Berlin: Mouton de Gruyter.

Sells, Peter. 1987. "Aspects of Logophoricity." Linguistic Inquiry 18: 445-79.

Stalnaker, Robert C. 1981. "Indexical Belief." Synthese 49: 129-51. Reprinted in: (Stalnaker 1999, 130-49) https://doi.org/10.1007/BF01063917

Stalnaker, Robert C. 1999. Context and Content. Oxford: Oxford University Press. https://doi.org/10.1093/0198237073.001.0001

Stalnaker, Robert C. 2008. Our Knowledge of the Internal World. Oxford: Oxford University Press. https://doi.org/10.1093/acprof:oso/9780199545995.001.0001

Sosa, Ernest. 1970. "Propositional Attitudes De Dicto and De Re." The Journal of Philosophy 67 (21): 883-96. https://doi.org/10.2307/2024040 
Textor Mark. 2007. "Frege's Theory of Hybrid Proper Names Developed and Defended." Mind 116 (464): 947-81. https://doi.org/10.1093/mind/fzm947

Textor, Mark. 2015. "Frege's Theory of Hybrid Proper Names Extended." Mind, 124 (495): 823-47. https://doi.org/10.1093/mind/fzv032

Weber, Clas. 2013. "Centered Communication." Philosophical Studies 166 (1): 20523. https://doi.org/10.1007/s11098-012-0066-6

Vendler, Zeno. 1967. Linguistics in Philosophy. Ithaca: Cornell University Press.

Verdejo, Victor. 2017. "Explaining Public Action." Topoi.

https://doi.org/10.1007/s11245-017-9524-2 